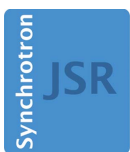

JOURNAL OF SYNCHROTRON RADIATION

ISSN 1600-5775

Received 27 June 2016

Accepted 21 November 2016

Edited by S. Svensson, Uppsala University, Sweden

Keywords: X-ray photoelectron spectroscopy; $\mathrm{X}$-ray photoelectron diffraction; scanning tunneling microscopy.

Supporting information: this article has supporting information at journals.iucr.org/s

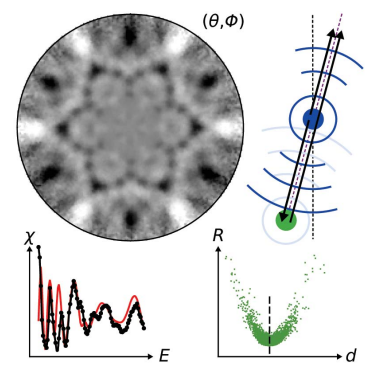

OPEN $\odot$ ACCESS

\section{Surface science at the PEARL beamline of the Swiss Light Source}

\author{
Matthias Muntwiler, ${ }^{\mathrm{a} *}$ Jun Zhang, ${ }^{\mathrm{a}}$ Roland Stania, ${ }^{\mathrm{a}, \mathrm{b}}$ Fumihiko Matsui, \\ Peter Oberta, ${ }^{\mathrm{a}, \mathrm{d}}$ Uwe Flechsig, ${ }^{\mathrm{a}}$ Luc Patthey, ${ }^{\mathrm{a}}$ Christoph Quitmann, ${ }^{\mathrm{a}, \mathrm{e}}$ \\ Thilo Glatzel, ${ }^{f}$ Roland Widmer, ${ }^{\mathrm{g}}$ Ernst Meyer, ${ }^{\mathrm{f}}$ Thomas A. Jung, ${ }^{\mathrm{a}, \mathrm{f}}$ \\ Philipp Aebi, ${ }^{\text {h }}$ Roman Fasel $^{\mathrm{g}}$ and Thomas Greber ${ }^{\mathrm{b}}$
}

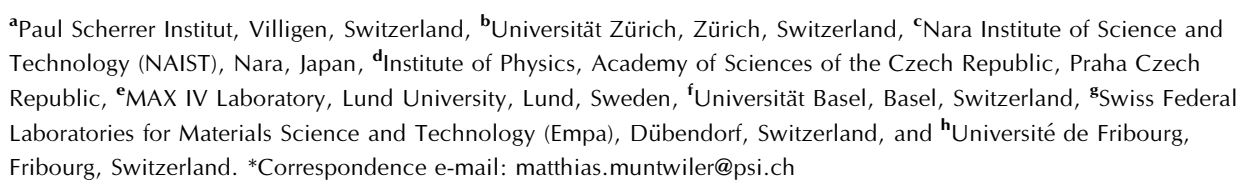

The Photo-Emission and Atomic Resolution Laboratory (PEARL) is a new soft X-ray beamline and surface science laboratory at the Swiss Light Source. PEARL is dedicated to the structural characterization of local bonding geometry at surfaces and interfaces of novel materials, in particular of molecular adsorbates, nanostructured surfaces, and surfaces of complex materials. The main experimental techniques are soft X-ray photoelectron spectroscopy, photoelectron diffraction, and scanning tunneling microscopy (STM). Photoelectron diffraction in angle-scanned mode measures bonding angles of atoms near the emitter atom, and thus allows the orientation of small molecules on a substrate to be determined. In energy scanned mode it measures the distance between the emitter and neighboring atoms; for example, between adsorbate and substrate. STM provides complementary, real-space information, and is particularly useful for comparing the sample quality with reference measurements. In this article, the key features and measured performance data of the beamline and the experimental station are presented. As scientific examples, the adsorbate-substrate distance in hexagonal boron nitride on $\mathrm{Ni}(111)$, surface quantum well states in a metal-organic network of dicyano-anthracene on $\mathrm{Cu}(111)$, and circular dichroism in the photoelectron diffraction of $\mathrm{Cu}(111)$ are discussed.

\section{Introduction}

\subsection{Scientific case}

Surface science is an active, interdisciplinary field with applications in chemistry and physics such as heterogeneous catalysis, energy conversion, semiconductor and molecular electronics, spintronics and quantum magnetism. In these applications, chemical bonding, electronic charge transfer and magnetic interactions at interfaces play an important role, and many of the effects are intimately coupled to the atomic structure at the interface. Therefore, knowing the detailed structure is essential for the understanding of the underlying physics, and for the development and testing of theoretical calculations.

The Photo-Emission and Atomic Resolution Laboratory (PEARL) is a new soft X-ray beamline and surface science laboratory at the Swiss Light Source (SLS). It has been designed by a consortium of Swiss research groups active in surface science for the study of local atomic geometry at the surface of a wide range of novel organic and inorganic systems. 
Such systems include, for example, functional organic molecules (Fasel et al., 1996; Muntwiler et al., 2005; Pawlak et al., 2012), supramolecular networks (Barth, 2007; Lobo-Checa et al., 2009; Zhang et al., 2014), molecular magnets (Scheybal et al., 2005), chiral recognition (Fasel et al., 2004; Greber et al., 2006; Schillinger et al., 2007), endohedral fullerenes (Treier et al., 2009; Westerström et al., 2012, 2014), ultrathin metal oxides (Jaouen et al., 2015), surfaces of ferroelectrics (Despont et al., 2006) and surface alloys (Corso et al., 2010; Pawlak et al., 2015).

Owing to the stringent requirements of ultra-high vacuum (UHV) in surface science experiments many of the mentioned samples have to be prepared in situ without breaking UHV conditions between preparation and measurement. It is therefore crucial to have standard analytical tools available which allow for a quick assessment of the sample quality before long-running measurements are made. Furthermore, it is important that complementary analytical methods can be applied on the same sample if the correlation between atomic structure and electronic or magnetic properties is investigated. At synchrotron-based facilities these requirements are particularly challenging since the focus on instrument development is often laid on one specific technique. At PEARL, the aspect of integrating a reasonably complete surface science laboratory at a synchrotron facility was the major design goal.

\subsection{Technical case}

In surface science, charged particles, mostly electrons, are typically used as probes in a variety of experimental methods because they are particularly surface sensitive. At PEARL, the main analytical methods are soft X-ray photoelectron spectroscopy (XPS), photoelectron diffraction (XPD/PhD) as well as scanning tunneling microscopy (STM) and spectroscopy (STS). Since the instrumentation for $\mathrm{XPD} / \mathrm{PhD}$ is mostly the same as for angle-resolved photoelectron spectroscopy (ARPES) in general, the facility also offers the spectroscopy of core-levels, valence bands (with limited resolution), Auger decays and resonant excitations. In the following, we will discuss mainly photoelectron diffraction and scanning tunneling microscopy.

Owing to their ease of use, scanning probe techniques have evolved to be popular methods for real-space imaging of surface structure and other surface properties at various length scales down to atomic resolution. However, vertical distance and angles between atoms are not easily accessible, and the contributions of the atomic and the electronic structure to a measured contrast are sometimes difficult to distinguish. Photoelectron diffraction is a technique to measure local atomic structure by exploiting the wave nature of photoelectrons and its diffractive properties at atomic potentials. Diffraction features appear as a variation of the photoelectron intensity as a function of emission angle and electron energy. By selecting a particular core-level photoemission peak, the technique is chemically selective, and due to a short mean free path it is sensitive to the topmost atomic layers. Photoelectron diffraction in angle-scanned mode
(XPD) (Fadley, 1984; Osterwalder et al., 1995; Fadley, 2010) is suitable for measuring bonding angles and the orientation of small molecules with respect to the substrate (Fasel et al., 1996); while in energy scanned mode, where the acronym $\mathrm{PhD}$ is more commonly used, it is sensitive to the distance between neighboring atoms, for example, between adsorbed molecules and the substrate (Woodruff, 2007).

Energy-scanned $\mathrm{PhD}$ requires a photon source with smoothly tunable energy in the soft X-ray range. Tunable photon energy is also beneficial in many other cases. In photoelectron spectroscopy (XPS), the photoionization yield and the probing depth depend on photon energy, and the surface/bulk ratio can be adjusted. By tuning the photon energy to a particular X-ray absorption line, the photoemission cross section can be resonantly enhanced (Treier et al., 2009), or a particular symmetry of the photoelectron wavefunction can be selected (Morscher et al., 2011; Matsui et al., 2015). Using the core-hole clock method with Raman-active Auger decays, ultrafast delocalization dynamics in the conduction band can be studied (Föhlisch et al., 2005; Jaouen et al., 2015). In angle-scanned XPD the ratio between forward and backward scattering can be tuned.

Fig. 1 illustrates the basic measurement principle of XPD in the hypothetical example of two atoms. Let us assume a nitrogen atom is bonded to a nickel atom below, and the connecting line of the two atoms is tilted by $15^{\circ}$ with respect to the reference axis (the sample normal in an actual system). This diatomic cluster is a reduced version of the h-BN/Ni system to be discussed in the Scientific highlights section below. At a fixed kinetic energy of $215 \mathrm{eV}$, the intensity of the $\mathrm{N} 1 s$ XPS peak exhibits the angular distribution shown in panel (a), as calculated using the EDAC electron diffraction code (García de Abajo et al., 2001). The angular distribution of the intensity is displayed in a stereographic projection: the polar coordinates of the unit hemisphere $(\theta, \varphi)$ are mapped to planar Cartesian coordinates according to $(x, y)=$ $2 \tan (\theta / 2)(\cos \varphi, \sin \varphi)$. The wavefunction of the photoelectron emanating from the emitter atom is scattered at the neighboring atom, and the direct and scattered waves interfere in the detector. The results are characteristic, circular interference fringes around the nearest-neighbor direction. Such features have been used in experiments to locate the adsorption site of atoms on metal surfaces, for example, in the case of $\mathrm{O} / \mathrm{Rh}(111)$ (Wider et al., 1998).

If we cut the angular distribution of the photoelectron intensity along the $90^{\circ}$ azimuth and expand the calculation in kinetic energy, we obtain the pattern shown in Fig. 1(c). The interference fringes appear again centered on the angle of the connecting line between the atoms. The frequency of the fringes is given by the distance between the two atoms, and the energy-dependent wavelength of the photoelectron. In the plot (note the logarithmic gray scale) it is obvious that the diffraction features typically correspond to a small variation of intensity on a strong but slowly varying background. Theoretically, this is due to the angular dependence of the photoemission cross section and the scattering factors. In the experiment, additional factors such as photon flux, sample 


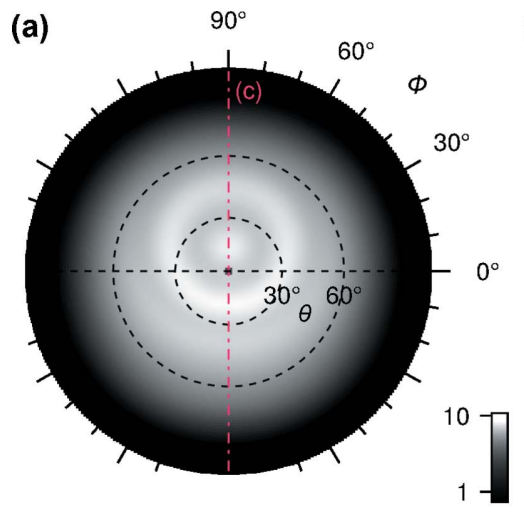

(b)
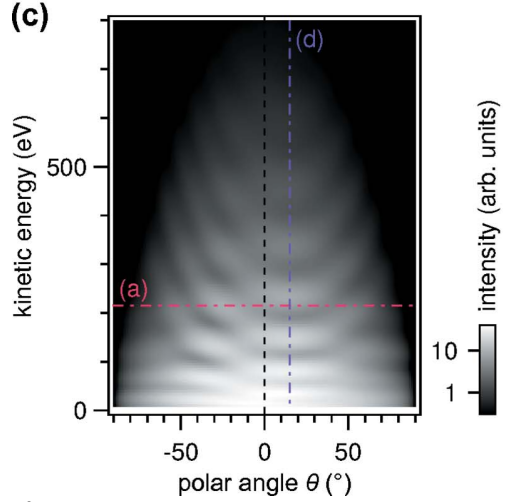

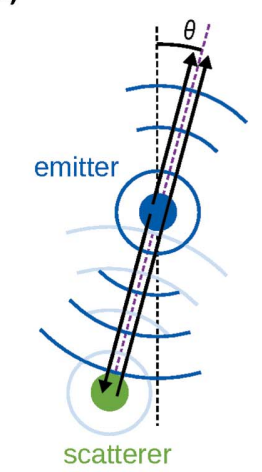

(d)

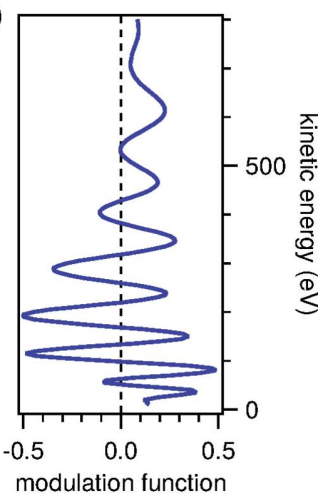

Figure 1

Calculated photoelectron diffraction versus angle and energy for a diatomic system consisting of a nitrogen atom as emitter and nickel as scatterer. (a) Hemispherical angle-distribution of the photoelectron intensity in stereographic projection. The gray scale is logarithmic. (b) Schematic electron scattering geometry. The nearest-neighbor direction is tilted by $15^{\circ}$ with respect to normal emission. The azimuthal angle is $\varphi=$ $90^{\circ}$. (c) Photoelectron intensity as a function of kinetic energy and polar angle $\theta$. Corresponding section lines to panels $(a)$ and $(d)$ are indicated. (d) One-dimensional modulation function extracted from panel (c) along the vertical line at $\theta=15^{\circ}$. A detailed description is given in the text.

orientation and detection efficiency may contribute, and are often difficult to separate from the diffraction signal. The relevant diffraction features are extracted by calculating the modulation function

$$
\chi=\frac{I-I_{0}}{I_{0}},
$$

where $I_{0}$ is a smooth function from a non-parametric fit of the data, such as a cubic spline or locally weighted linear regression (Woodruff, 2007). The modulation function of the present data set near $\theta=15^{\circ}$ is shown in Fig. $1(d)$. Experiment and theory can be compared quantitatively by calculating the Pendry $R$-factor of the modulation functions (Woodruff, 2007),

$$
R=\frac{\sum\left(\chi_{\exp }-\chi_{\text {theo }}\right)^{2}}{\sum\left(\chi_{\exp }^{2}+\chi_{\text {theo }}^{2}\right)} .
$$

Conventionally, $\mathrm{PhD}$ scans were measured as one-dimensional line scans similar to the profile in Fig. $1(d)$. However, modern angle-dispersive analysers make it easy to measure multiple directions in parallel as in Fig. 1(c). This has two advantages

Table 1

Specifications of the X-ray optics.

\begin{tabular}{ll}
\hline Source type & Bending magnet \\
Mirrors & $2 \times$ toroidal \\
Monochromator & Planar grating, uncollimated beam \\
Gratings & 600 and 1200 lines $\mathrm{mm}^{-1}$ \\
Energy range & $60-2000 \mathrm{eV}$ \\
Beam size (collimated) & $190 \mu \mathrm{m} \times 70 \mu \mathrm{m}$ \\
Beam size (uncollimated) & $1100 \mu \mathrm{m} \times 1300 \mu \mathrm{m}$ \\
Maximum flux & $2 \times 10^{11}$ photons s \\
Photon energy at maximum flux & $800 \mathrm{eV}$ \\
Ultimate energy resolution $(E / \Delta E)$ & 7000 \\
Polarization modes & Linear horizontal, elliptical (left/right) \\
\hline
\end{tabular}

over line scans. First, the precise emission angle can be determined from the same dataset as the distance between emitter and scatterer. Second, if the data contain diffraction features from multiple scattering configurations, correspondingly, more structural parameters can be determined at once.

\section{Technical setup}

\subsection{Beamline optics}

The specifications for the X-ray optics are based on the scientific and technical case described above. The details of the optical design have been discussed in a previous article (Oberta et al., 2011). Essentially, the beamline covers the photon energy range from 60 to $2000 \mathrm{eV}$. It is optimized for high photon flux in the range between 500 and $1000 \mathrm{eV}$ where most photoelectron diffraction measurements of the lighter elements take place. Higher photon energies give access to resonant excitation of $4 f$ levels in rare earths, and the low end allows for basic spectroscopy of the valence region. The key figures are summarized in Table 1.

The beamline is installed at a $1.4 \mathrm{~T}$ bending magnet which delivers a smooth photon spectrum with a critical energy of $5 \mathrm{keV}$. The main polarization mode of the bending magnet is linear horizontal. By tilting the trajectory of the stored electron beam, the polarization can be switched to elliptical. The optical layout is based on a plane-grating monochromator (Petersen et al., 1995) operating in non-collimated light and negative diffraction order, as shown schematically in Fig. 2. This scheme provides a good compromise between high photon flux and high energy resolution. In the optimum energy range, it allows to distinguish chemically shifted core levels or spin multiplets of the order of 0.1 to $0.5 \mathrm{eV}$. For systems where high resolution is not needed, it is possible to trade resolution for flux. Compared with other soft X-ray beamlines at the SLS operating in collimated light (Strocov et al., 2010; Piamonteze et al., 2012), the number of reflecting surfaces is reduced by one to save photon flux. As a drawback, the fixed-focus condition is set by design and cannot be modified during operation, giving the user less control over the harmonics in the spectrum. Using two selectable diffraction gratings (600 and 1200 lines $\mathrm{mm}^{-1}$ ), photon energy is smoothly tunable in two overlapping energy ranges (60-1100 and $200-2000 \mathrm{eV}$, respectively). 

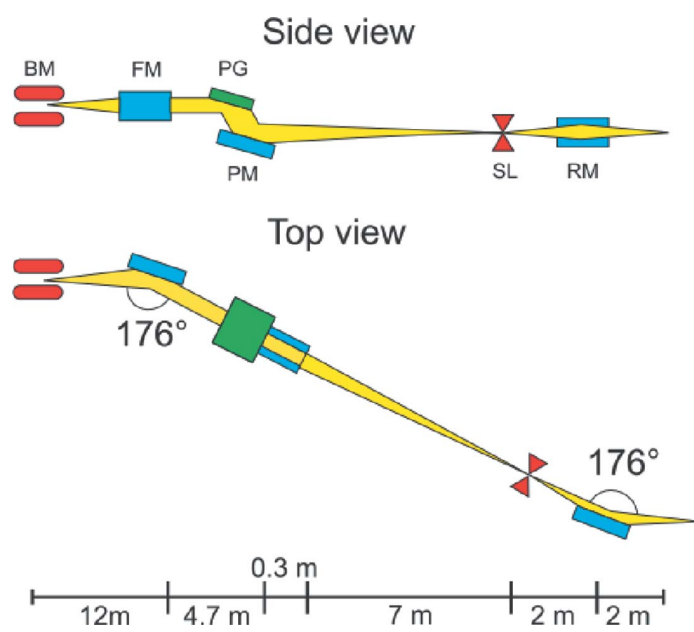

Figure 2

Schematic layout of the beamline, showing the optical path of the X-rays from the bending magnet to the endstation. The principal optical elements are: bending magnet (BM), focusing mirror (FM), plane grating (PG), plane mirror (PM), exit slit (SL), refocusing mirror (RM). Reprinted from Oberta et al. (2011) with permission from Elsevier.

Although the signal-to-noise ratio in photoelectron diffraction can benefit strongly from high photon flux, many samples, particularly organic molecules, are susceptible to radiation damage if the flux is too high. To mitigate the problem, it is not sufficient to reduce the photon flux because that reduction would have to be compensated by increased exposure time. Rather, high electron yield with high conver-

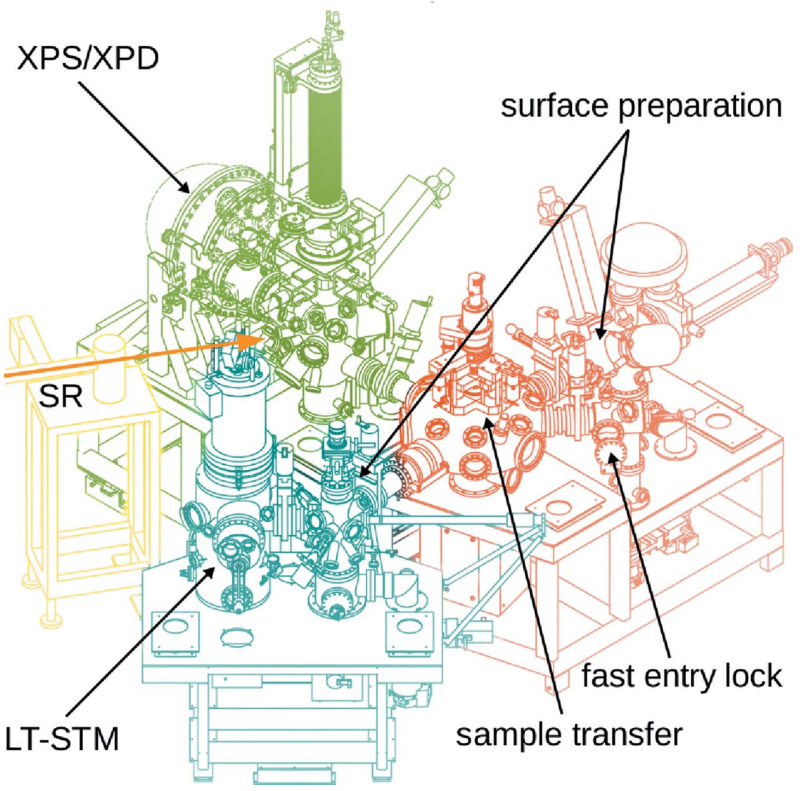

Figure 3

Conceptual rendering of the endstation. The three substations for angleresolved photoelectron spectroscopy and diffraction (XPS/XPD, green), scanning tunneling microscopy (LT-STM, blue) and surface preparation (red) are connected to a central, rotary sample transfer under UHV. The synchrotron radiation (SR) enters the XPS/XPD station along the path marked by an arrow. The drawing does not accurately represent the installation status of auxiliary devices.
Table 2

Specifications of the surface preparation system.

\begin{tabular}{ll}
\hline Base pressure & $4 \times 10^{-10} \mathrm{mbar}$ \\
Sample cleaning & Ar ion sputtering \\
Heating, radiative & $1200 \mathrm{~K}(100 \mathrm{~W})$ \\
Heating, direct current & $12 \mathrm{~A}, 60 \mathrm{~V}$ \\
Heating, e-beam & $1500 \mathrm{~K}$, under construction \\
Temperature measurement, infrared & $625-1575 \mathrm{~K}$ \\
Cooling & $40 \mathrm{~K}(\mathrm{LHe}), 77 \mathrm{~K}$ (LN2) \\
Organic evaporator & Six crucibles, up to 900 K \\
Gated user ports & $2 \times$ DN40CF \\
LEED/Auger & Omicron SpectaLEED \\
Residual gas analysis & $0-200$ a.m.u. \\
Gas dosing & Leak valve \\
Vapor deposition & Leak valve \\
Load lock & $1 \times 10^{-7}$ mbar \\
Sample transfer & Four spaces \\
Sample storage & 22 spaces $\left(2 \times 10^{-10}\right.$ mbar) \\
\hline
\end{tabular}

sion and detection efficiency is required. For the one part this is achieved with tunable photon energy, as the photoionization cross section can vary by orders of magnitude over the energy range of soft X-rays. For the other part, the photon flux can be spread over a larger area of the sample, thereby reducing the flux density, while the entire illuminated sample area is seen by the detector. At PEARL, the refocusing mirror unit can be switched to produce either a focused or a defocused beam on the sample.

\subsection{Experimental station}

The experimental station is divided into three sub-systems (Fig. 3): one (attached to the beamline) for the photoemission measurements, one for scanning tunneling microscopy and one for surface preparation. All processes and measurements take place in UHV at a base pressure below $2 \times 10^{-10} \mathrm{mbar}$. The sub-systems are connected by a reliable in situ sample transfer system. Though the system operates at room temperature, the transfer time between measurement positions is short enough to prevent cold samples (below $100 \mathrm{~K}$ initially) from heating up above $200 \mathrm{~K}$. Samples and organic powders for evaporation are introduced from ambient or a UHV suitcase via a fast-entry lock.

The sample preparation system provides standard surface science techniques for preparation (ion bombardment, annealing by radiative heating) and characterization [low energy electron diffraction (LEED), Auger electron spectroscopy, residual gas analysis]. A sixfold array of molecular beam evaporators with in situ exchangeable quartz crucibles for materials which sublimate below $900 \mathrm{~K}$ is available, as well as gated ports for user-supplied evaporators. A hightemperature annealing stage $(1500 \mathrm{~K})$ is under construction. For full specifications, see Table 2.

The low-temperature STM (Omicron Nanotechnology $\mathrm{GmbH}$, Table 3) provides real-space sample characterization down to atomic resolution. Standard topography mode allows for quick assessment of the surface quality and reference to measurements at the user's home laboratory, $\mathrm{d} I / \mathrm{d} V$ spectroscopy and mapping can be used to measure the local density of states near the Fermi level. Thanks to careful damping inside 
Table 3

Specifications of the scanning probe microscopy system.

\begin{tabular}{ll}
\hline Base pressure & $1 \times 10^{-10}$ mbar \\
STM & Omicron LT-STM, Matrix electronics \\
Detection & Tunneling current \\
Operating temperature & $4.2,77,298 \mathrm{~K}$ \\
Gas dosing & $\mathrm{Xe} / \mathrm{CO}$ in situ \\
\hline
\end{tabular}

and outside the chamber, the STM has proven insusceptible to vibrations and acoustic noise from the synchrotron environment.

The photoemission station is designed as a state-of-the-art ARPES facility with a 'Carving 2.0' six-axis manipulator designed by PSI and Amsterdam University, and a Scienta EW4000 hemispherical electron analyser with two-dimensional detection. The specifications are summarized in Table 4 . The measurement geometry is illustrated in Fig. 4(a). The entrance lens stack of the analyser is at a fixed angle $\beta=60^{\circ}$ with respect to the incoming synchrotron light. The entrance slit of the analyser is oriented vertically (parallel to the main axis of rotation). In this orientation, the symmetry of the differential photoemission cross section with respect to the light polarization allows for a homogeneous illumination of the detector.

The primary rotation axis is the polar rotation $\theta$ about the $z$ axis. The secondary rotation axes are the tilt $\psi$ about the $y^{\prime}$ axis, and the azimuthal rotation $\varphi$ about the surface normal n of the sample. The new version 2.0 of the Carving manipulator features an improved bearing concept of the primary rotation to reduce the sphere of confusion: three-dimensional mechanical test measurements after assembly show that, under polar rotation $\theta$, the sample moves by less than $25 \mu \mathrm{m}$ in the scattering ( $x y)$ plane and less than $65 \mu \mathrm{m}$ along the $z$ axis. For the secondary rotation axes $\psi$ and $\varphi$, the displacement is less than $25 \mu \mathrm{m}$. Such high mechanical precision is essential for angle-scanned measurements due to the small beam size and the small focal depth of the analyser, either of which is of the order of $100 \mu \mathrm{m}$. To take advantage of the high precision, however, the sample must be mounted with the same precision on the sample plate so that the surface is aligned with the rotation center of the manipulator. Usually, this requires a precise optical survey of the shape of the specimen and the manufacturing of a sample holder that is tailored to the specific shape.

The sample can be cooled down to $35 \mathrm{~K}$ using liquid helium (LHe). The actual sample temperature was confirmed by adsorption and desorption of argon on a $\mathrm{Cu}(111)$ surface, compared with literature values of the desorption temperature (Berthold et al., 2004; Meyer et al., 2008). Due to the particular design of the Carving manipulator, thermal contraction of the primary axis due to cryogenic cooling is negligible.

The EW4000 electron analyser contains a two-dimensional multi-channel plate detector where one axis corresponds to the kinetic energy of the electron and the other axis to the emission angle $\alpha$. The nominal acceptance angle of this detector is $60^{\circ}$. In practice, transmission and matrix element effects limit the useful range to about $50^{\circ}$. Combining the
Table 4

Specifications of the photoelectron spectroscopy system.

\begin{tabular}{ll}
\hline Base pressure & $2 \times 10^{-10} \mathrm{mbar}$ \\
Detector type & Photoelectron spectrometer \\
Detector model & Scienta EW4000 \\
Energy resolution $E_{\text {pass }} / \Delta E$ & 1750 \\
Angle resolution & $0.5^{\circ}$ \\
Manipulator & Carving 2.0 \\
Goniometer & Three translations, three rotations \\
Polar rotation & $0^{\circ}$ to $180^{\circ}\left(60^{\circ}=\right.$ normal incidence) \\
Tilt rotation & $-28^{\circ}$ to $+28^{\circ}$ \\
Azimuthal rotation & $-180^{\circ}$ to $+180^{\circ}$ \\
Cooling & $35 \mathrm{~K}$ \\
Heating, radiative & $400 \mathrm{~K}$ \\
Sample mounting & Omicron-style sample plate \\
Photon flux monitoring & Photocurrent on Pt mirror \\
& Photocurrent on Au mesh \\
\hline
\end{tabular}
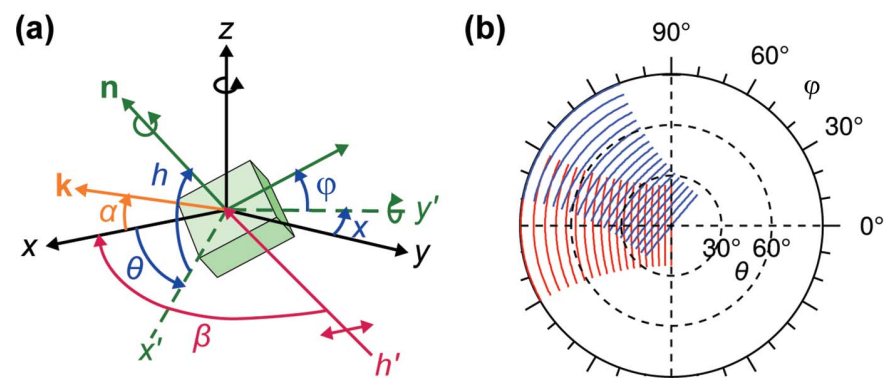

Figure 4

(a) Measurement geometry in the ARPES chamber. The coordinate axes $x, y$ and $z$ are fixed in the laboratory frame of reference. Photoelectrons are detected in the $x z$ plane under the acceptance angle $-30^{\circ} \leq \alpha \leq+30^{\circ}$ centered on the $x$ axis. The synchrotron beam enters at an angle $\bar{\beta}=60^{\circ}$ with respect to the $x$ axis, the polarization vector of horizontal light is in the $x y$ plane. The sample can be moved in the $x, y$ and $z$ directions, and rotated about the $z$ (polar angle $\theta$ ) and $y^{\prime}$ (tilt angle $\psi$ ) axes, as well as about the surface normal $\mathbf{n}$ (azimuthal angle $\varphi$ ). (b) Scanning scheme of angle-scanned photoelectron diffraction in the spherical coordinate system in stereographic projection. A full scan of emission angles in the hemisphere is a combination of polar $(\theta)$ and azimuthal $(\varphi)$ scans. Each of the curved lines in the plot corresponds to the angle range detected in one shot. For clarity, only a few angles are shown.

manipulator and detector angles, photoelectron counts are collected as a function of the four angles $\theta, \psi, \varphi$ and $\alpha$. However, in presentation graphs, angle-scanned photoelectron diffraction data are typically displayed in the spherical coordinate system $\left(\theta_{\mathrm{s}}, \varphi_{\mathrm{s}}\right)$ of the sample as in Fig. 1(a). Assuming that the angular dependence of the matrix element can be neglected (e.g. by normalization), instrument coordinates are mapped to sample coordinates by applying a series of rotations to the Cartesian vector $\mathbf{k}_{\alpha}=(\cos \alpha, 0, \sin \alpha)$ which marks the detection angle in the laboratory frame of reference (Greif et al., 2014). In the sample frame, the emission vector $\mathbf{k}_{\mathrm{s}}$ becomes, thus

$$
\mathbf{k}_{\mathrm{s}}=R_{x}^{-1}(\varphi+\pi / 2) R_{z}^{-1}(\theta) R_{y}^{-1}(\psi) \mathbf{k}_{\alpha},
$$

where $R_{x}^{-1}, R_{y}^{-1}$ and $R_{z}^{-1}$ denote the inverse rotation matrices about the coordinate axes $x, y$ and $z$, respectively. $\mathbf{k}_{\mathrm{s}}$ can then be mapped to spherical coordinates in the canonical way taking the $x$ axis as the surface normal. Fig. 4(b) shows the lines accepted by the analyser for a number of manipulator 
positions and how they map to the sample frame according to equation (3). On the unit hemisphere, each of the lines corresponds to an arc of a great circle. In the stereographic projection, it appears curved with a $\theta$-dependent curvature. A full hemispherical diffractogram can be measured by combined $(\theta, \varphi)$ scans with a step size of $1^{\circ}$ or $2^{\circ}$ for $\theta$, and between $15^{\circ}$ and $50^{\circ}$ for $\varphi$ in typically 6 to $24 \mathrm{~h}$, depending on the signal and desired amount of oversampling.

\section{Measured performance}

\subsection{Photon flux}

The photon flux is measured by a calibrated silicon diode after the refocusing mirror, Fig. 5. The two laminar diffraction gratings cover an energy range from $60 \mathrm{eV}$ up to $2000 \mathrm{eV}$ with an overlapping region between 100 and $1000 \mathrm{eV}$. The 600 lines $\mathrm{mm}^{-1}$ grating is optimized for high photon flux, whereas the 1200 lines $\mathrm{mm}^{-1}$ grating is required for photon energies above $1000 \mathrm{eV}$, or for better energy resolution below $1000 \mathrm{eV}$. The dashed and solid lines mark the practical lower and upper limits which can be set by the front-end aperture, respectively, at a typical exit slit aperture of $100 \mu \mathrm{m}$. The upper limit is given by the physical size of the focusing mirror. The results are summarized in Table 5.

\subsection{Energy resolution}

The energy resolution in photoelectron spectroscopy is limited by the beamline optics and the electron analyser. In this section, we first demonstrate the ultimate resolution of the optics by measuring gas phase X-ray absorption spectra of nitrogen. Second, we discuss the energy resolution of the complete system derived from photoelectron spectra under typical measurement conditions. In most practical cases, it is necessary to find a compromise between energy resolution and count rate by opening the apertures of the beamline and the analyser.

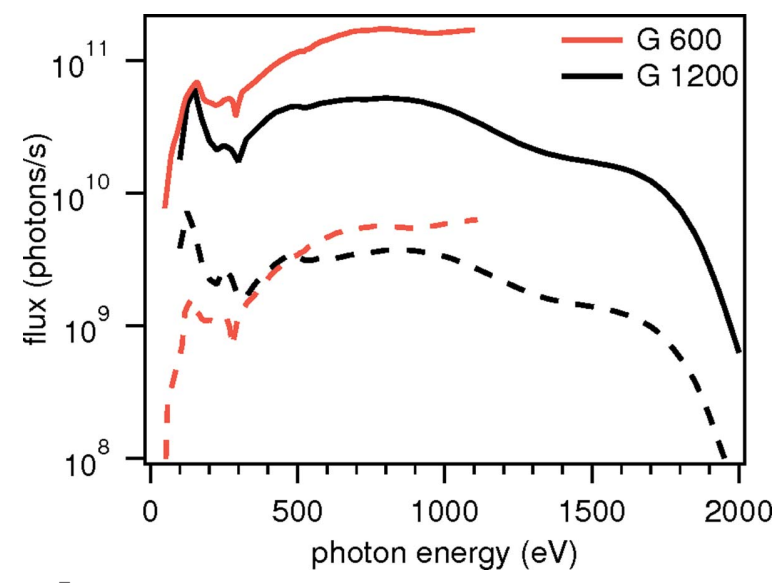

Figure 5

Photon flux after the refocusing mirror measured on a calibrated silicon photodiode for the 600 and 1200 lines $\mathrm{mm}^{-1}$ diffraction gratings. The solid and broken lines were measured for maximum $(720 \mu \mathrm{rad} \times$ $1200 \mu \mathrm{rad})$ and minimum $(120 \mu \mathrm{rad} \times 120 \mu \mathrm{rad})$ front-end aperture, respectively. The exit slit size is $100 \mu \mathrm{m}$.
Table 5

Summary of measured performance values of the beamline optics.

Maximum photon flux is measured at $h v=800 \mathrm{eV}$, energy resolution at $400 \mathrm{eV}$. $\mathrm{G} 600$ (G 1200) denotes the 600 lines $\mathrm{mm}^{-1}$ (1200 lines $\left.\mathrm{mm}^{-1}\right)$ grating. See text and supporting information for details

\begin{tabular}{llll}
\hline Property & Unit & G 600 & G 1200 \\
\hline Maximum photon flux & photons s $^{-1}$ & $1.7 \times 10^{11}$ & $5.2 \times 10^{10}$ \\
Photon flux (small aperture) & photons s $^{-1}$ & $5.6 \times 10^{9}$ & $3.7 \times 10^{9}$ \\
Resolving power (XAS) & & 5550 & 6860 \\
Energy resolution (XAS) & $\mathrm{meV}$ & 72 & 58 \\
Energy resolution (XPS) & $\mathrm{meV}$ & 103 & 98 \\
\hline
\end{tabular}

Total ion yield gas phase spectra are measured in a gas cell installed after the exit slit of the monochromator. The apertures are set at the lowest practical values of $1 \mathrm{~mm} \times 1 \mathrm{~mm}$ for the front-end, corresponding to an acceptance angle of $(120 \mu \mathrm{rad})^{2}$, and $50 \mu \mathrm{m}$ for the exit slit. The measured $\mathrm{N}_{2}$ $1 s-\pi^{*}$ spectra are plotted in Fig. 6. The ratio of total yield between the first valley at $400.8 \mathrm{eV}$ and the third peak at $401.2 \mathrm{eV}$ is a sensitive measure of the overall energy resolution where lower values indicate better energy resolution (Chen \& Sette, 1989). The advantage of the valley-to-peak (v/p) ratio over curve fitting is that it is independent of the calibration of the energy scale and less susceptible to correlations between fit parameters. For a quantitative measure, the spectrum is modeled with the sum of seven Voigt profiles and $\mathrm{v} / \mathrm{p}$ is compared with the measurement. The basic parameters for the model spectrum, the natural line width of $113 \mathrm{meV}$ FWHM and peak positions, are taken from the literature (Kato et al., 2007). The resulting values for the resolving power are 5550 and 6860 for the 600 lines $\mathrm{mm}^{-1}$ and 1200 lines $\mathrm{mm}^{-1}$ gratings, respectively, very close to the corresponding values 5500 and 7000 from the design calculations (Oberta et al., 2011). Benchmark values are summarized in Table 5. Detailed results and an additional discussion of curve fits are given in the supporting information.

To check the energy resolution of the complete system we measure the width of the Fermi edge of a polycrystalline gold
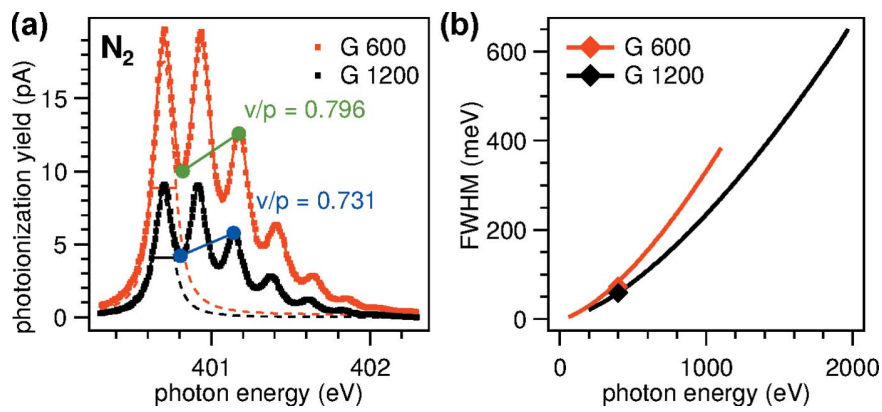

Figure 6

(a) Total yield absorption spectra of nitrogen measured with monochromatic light from the 600 lines $\mathrm{mm}^{-1}$ grating (G 600) and the 1200 lines $\mathrm{mm}^{-1}$ grating (G 1200), at an exit slit setting of $50 \mu \mathrm{m}$. Solid lines are least-squares fits of Voigt functions as described in the text, dashed lines show the decomposed lowest-energy peak. The intensity ratio between the first valley and third peak (v/p) is indicated. (b) Calculated energy resolution of the beamline optics at the aperture settings of the nitrogen spectra. Experimental values from nitrogen XAS are marked. 

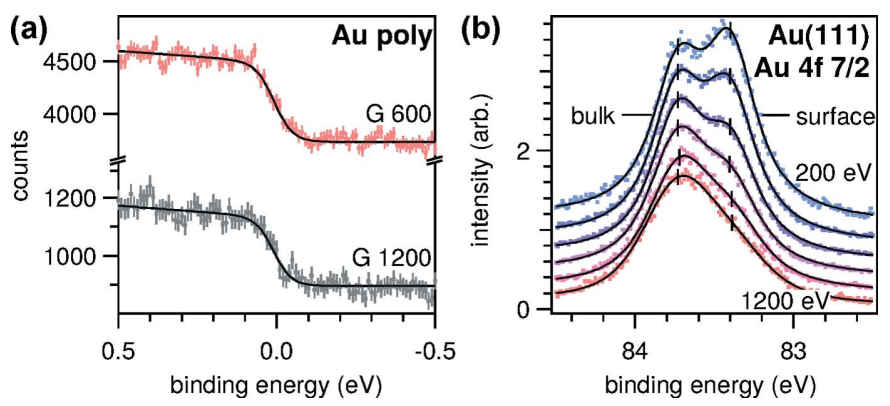

Figure 7

(a) High-resolution XPS spectra at the Fermi edge of polycrystalline gold measured at $h v=400 \mathrm{eV}$. Dots are electron counts integrated over the $60^{\circ}$ acceptance angle of the analyser with error bars estimated according to the Poisson distribution. Lines are curve fits of a Fermi function assuming, to first order, a linear increase of the density of states below $E_{\mathrm{F}}$. $(b)$ Highresolution XPS spectra of the $\mathrm{Au} 4 f_{7 / 2}$ peak of a single-crystal $\mathrm{Au}(111)$ surface measured at a series of photon energies between 200 and $1200 \mathrm{eV}$. Solid lines are curve fits of two Voigt profiles. The weighted mean of the binding energy of the bulk peak is $E_{\mathrm{B}}=(83.73 \pm 0.01) \mathrm{eV}$, and the surface core-level shift is $(0.329 \pm 0.001) \mathrm{eV}$. The spectra are normalized to the area of the bulk peak and vertically offset for clarity.

sample with XPS. The spectra in Fig. 7 are taken at essentially the same beamline settings as the nitrogen absorption spectra except that the front-end aperture is widened to $(240 \mu \mathrm{rad})^{2}$ to increase the count rate. The effect of the wider aperture on the energy resolution is less than $5 \%$ as confirmed in separate XAS measurements of nitrogen. The width of the Fermi edge contains two components, the intrinsic thermal broadening of the electron distribution in the material and the instrumental broadening by the analyser and the beamline. To first order, these effects add up quadratically as discussed in the supporting information (Kreutz et al., 1998). To reduce the first contribution as much as possible, we cool the sample to $40 \mathrm{~K}$. The spectra can be fit with a Fermi-Dirac distribution at $T=(341 \pm 40) \mathrm{K}$ and $T=(325 \pm 62) \mathrm{K}$, which amounts to a total instrumental broadening of 103 and $98 \mathrm{meV}$, respectively. Given the resolution of the X-ray optics discussed before, the analyser resolution is estimated to be $(76 \pm 17) \mathrm{meV}$ at the selected entrance slit $(0.2 \mathrm{~mm})$ and pass energy $(50 \mathrm{eV})$. Though the resolution of the analyser could be improved by lowering the pass energy, the low count rate due to the very low photoemission cross section of the valence band in the soft $\mathrm{X}$-ray regime did not allow so as the acquisition of each displayed spectrum took about $12 \mathrm{~h}$. On the other hand, the spectrum of an intense peak such as the $4 f_{7 / 2}$ core level resolving the surface core-level shift as in Fig. 7(b) can be acquired with the same high-resolution settings in less than $10 \mathrm{~min}$. These measurements demonstrate that the beamline is capable of resolving chemical shifts of core-levels of the order of $100 \mathrm{meV}$. However, at very high resolution and for low cross-section transitions, the count rate is limited.

\subsection{Spot size}

The spot size on the sample is a result of the size of the electron beam in the bending magnet, the optical magnification, aberrations and manufacturing tolerances. These effects sum up to a theoretical minimum spot size of $170 \mu \mathrm{m} \times 73 \mu \mathrm{m}$ on the sample as predicted by ray-tracing calculations (Oberta et al., 2011). Experimentally, the beam profile is measured on a scintillator plate at the nominal focus position of the refocusing mirror, $c f$. Fig. 8. The results for the small spot geometry, panel $(a)$, agree very well with the calculations. The minimum FWHM spot size observed is $190 \mu \mathrm{m} \times 70 \mu \mathrm{m}$ at a photon energy of $1000 \mathrm{eV}$ and a vertical exit slit aperture of $100 \mu \mathrm{m}$. The small spot is almost independent of the front-end aperture and the photon energy. Its horizontal width increases slightly towards lower photon energy $(230 \mu \mathrm{m}$ at $h v=400 \mathrm{eV})$.

The large spot setting of the refocusing mirror is designed to produce a convergent beam with an image distance of $5.7 \mathrm{~m}$ in the meridional (horizontal) plane, and a divergent beam with an image distance of $-28.8 \mathrm{~m}$ in the sagittal plane. The observed spot size depends significantly on the front-end acceptance, varying from $180 \mu \mathrm{m} \times 160 \mu \mathrm{m}$ at the smallest aperture (not shown) to $1.1 \mathrm{~mm} \times 1.3 \mathrm{~mm}$ at the maximum aperture [panel $(b)$ ].

Since the electron optics of the EW4000 analyser is optimized for a small spot of $100 \mu \mathrm{m}$, it is interesting to check the effect of the spot size on the angle and energy resolution of the analyser. For this, we measure the Shockley surface state on $\mathrm{Cu}(111)$ at a photon energy of $70 \mathrm{eV}$ [Figs. $8(c)$ and $8(d)$ ]. High-resolution measurements of this system are available in the literature (Reinert et al., 2001). It is obvious that in the large spot configuration the angle distribution is broader than
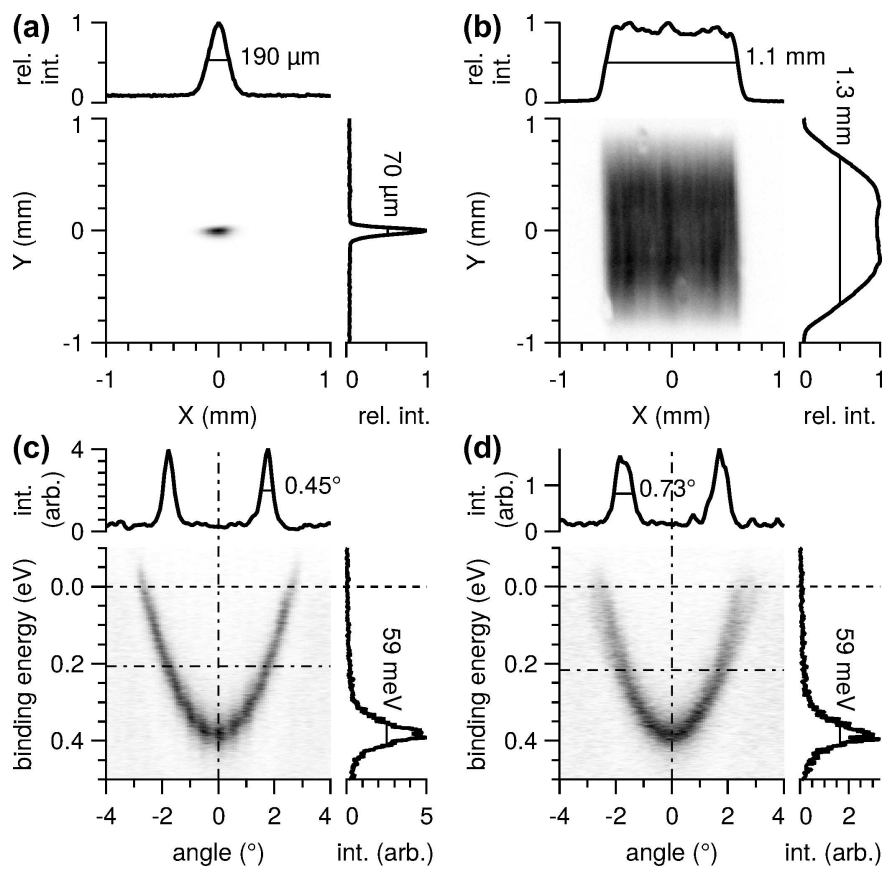

Figure 8

Small spot $(a, c)$ versus large spot $(b, d)$ setting of the refocusing mirror. $(a, b)$ Distribution of X-ray flux on a scintillator plate at normal incidence at the designated sample position. $(c, d)$ ARPES measurements of the Shockley state of the $\mathrm{Cu}(111)$ surface at $h v=70 \mathrm{eV}$. The measurements were taken in fixed-energy mode at a pass energy of $10 \mathrm{eV}$ and integrated over $10 \mathrm{~min}$. The line graphs show the integrals over one dimension $(a, b)$, or the profiles along the dash-dotted lines $(c, d)$, respectively. Full width at half-maximum is indicated. 
in the small spot configuration where the peak width approaches the nominal angle resolution of the analyser which is limited by the entrance mesh of the wide-angle lens. The influence of the spot size on the energy resolution is not obvious. In either case, the line width at the apex of the dispersion curve is $59 \mathrm{meV}$, limited by the instrumental energy resolution used in these measurements. We also find that the electron count rate is about $26 \%$ lower for the large spot while the total yield $(71 \mathrm{pA})$ does not change. Because this loss of electron counts has to be compensated by longer integration time, the advantage of the large spot (longer protection against radiation damage due to lower flux density), is reduced from the original ratio of beam size to about a factor 80 .

\section{Scientific highlights}

\subsection{Measuring adsorbate-substrate distance in boron nitride}

As a scientific example, we show angle- and energy-scanned photoelectron diffraction of hexagonal boron nitride (h-BN) on a $\mathrm{Ni}(111)$ surface. $\mathrm{h}-\mathrm{BN}$ is a well known atomically thin insulating layer that can be used to chemically and electronically decouple molecular adsorbates from the underlying metal (Muntwiler et al., 2005). The atomic structure of h-BN/ $\mathrm{Ni}(111)$ has been studied by low-energy electron diffraction, angle-resolved photoelectron diffraction, scanning tunneling microscopy, and density functional theory in the past (Gamou et al., 1997; Auwärter et al., 1999; Muntwiler et al., 2001; Grad et al., 2003). h-BN forms a commensurate $1 \times 1$ overlayer with a nitrogen atom at the top site and a boron atom at the f.c.c. hollow site, $c f$. Figs. $9(a)$ and $9(b)$. The layer is slightly corrugated due to a lattice mismatch and different bonding of nitrogen and boron to the substrate. The distance between the h-BN layer and the substrate, $d_{\mathrm{A}-\mathrm{S}}$, was studied by LEED previously and reported as $2.22 \AA$ (Gamou et al., 1997). A later angle-resolved XPD study reported a different value of $1.95 \AA$ (Muntwiler et al., 2001). Here, we look for an independent result using energy-scanned $\mathrm{PhD}$ in the backscattering configuration.

Angle-resolved XPD has the advantage that directions of atomic bonds can often be identified rather easily in a stereographic mapping of the photoelectron intensity without the need for a calculation. Such a map also helps to find the correct manipulator position with respect to specific diffraction features or bond directions for subsequent spectroscopy or $\mathrm{PhD}$ measurements. The diffraction pattern of the $\mathrm{N} 1 s$ peak of h-BN in Fig. $9(c)$ is assembled from XPS spectra measured at 2148 angular settings according to the procedure described in the supporting information. The polar angle dependence of the data is removed by normalization (see below), and a threefold average is applied according to the symmetry of the substrate. The diffraction pattern shows notable rings at $\theta>60^{\circ}$ that are centered on the nitrogenboron $(\mathrm{N}-\mathrm{B})$ and the nitrogen-nitrogen $(\mathrm{N}-\mathrm{N})$ nearestneighbor directions. In contrast to earlier published data (Auwärter et al., 1999), the pattern in Fig. 9(c) is sixfold symmetric due to the presence of two domains rotated by $180^{\circ}$

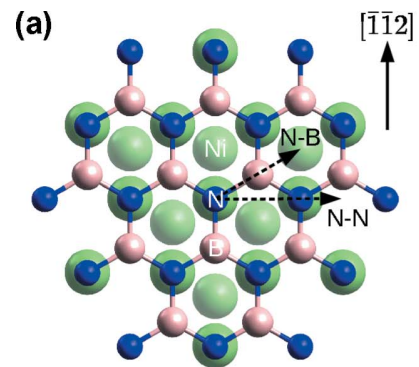

(c)
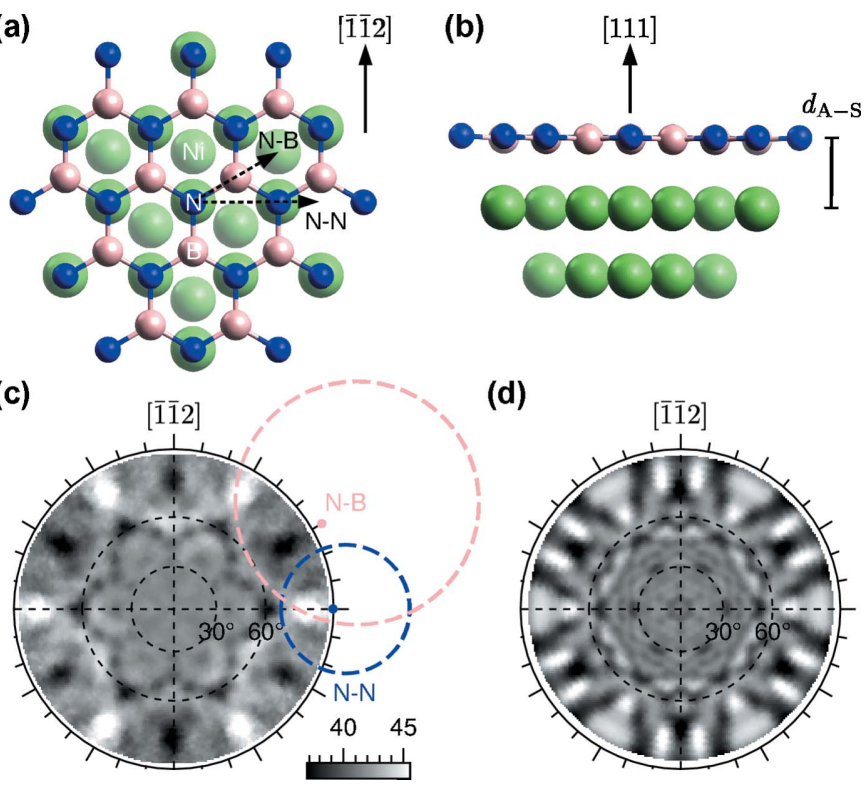

(d)

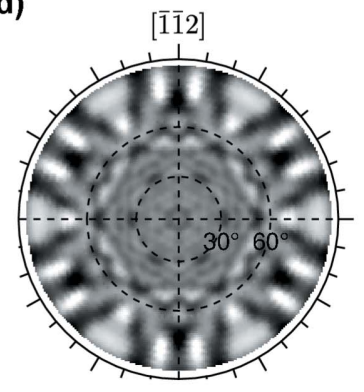

Figure 9

Angle-resolved photoelectron diffraction of the $\mathrm{N} 1 s$ peak of h-BN/ $\mathrm{Ni}(111)$ at $E_{\text {kin }}=399 \mathrm{eV}$. $(a, b)$ Cluster of atoms used in calculations in $(a)$ top view and $(b)$ side view. (c) Processed experimental data. The raw data consist of short XPS spectra $(11 \mathrm{~s}$ each) measured at 2148 angular settings distributed over the hemisphere ( $15^{\circ}$ steps in $\varphi$ and $1^{\circ}$ steps in $\theta$ ). The hemispherical diffractogram is assembled from $50^{\circ}$ wide detector images, normalized, and three-fold averaged as described in the text and the supporting information. The diffraction cones from scattering along the $\mathrm{N}-\mathrm{N}$ and $\mathrm{N}-\mathrm{B}$ nearest-neighbor directions [corresponding to the dashed arrows in panel $(a)$ ] are marked by dashed circles. (d) Calculated diffractogram from the best-fit structural model. The pattern is twofold averaged to match the symmetry of the measurement.

with respect to each other. It is known that, in addition to the most stable N-top, B-f.c.c. adsorption configuration, an N-top, B-h.c.p. configuration with a slightly lower binding energy can grow depending on a subtle difference in the quality of the substrate (Auwärter et al., 2003; Grad et al., 2003). The Ni crystal used in the present experiment was newly procured, and had not undergone the same number of cleaning steps as the one in the previous studies. Panel $(d)$ shows the corresponding simulation using the $E D A C$ multiple-scattering code (García de Abajo et al., 2001). The cluster in Figs. 9(a) and $9(b)$, showing only the most stable structure, is based on the optimized structural parameters discussed below. Qualitatively, measurement and calculation show the same diffraction features. However, a shift of features at higher polar angles indicates that the refraction at the surface may not be accurately described in the model.

To determine the adsorbate-substrate distance $d_{\mathrm{A}-\mathrm{S}}$ between the $\mathrm{N}$ and top-layer $\mathrm{Ni}$ atoms, the $\mathrm{PhD}$ intensity modulation of the $\mathrm{N} 1 s$ peak is measured as a function of electron energy and simulated numerically using the $E D A C$ code. In the simulations, seven structural and non-structural parameters are optimized using a particle swarm global search algorithm (Duncan et al., 2012) which minimizes the Pendry $R$-factor, equation (2). The optimized parameters are the adsorbate-substrate distance $d_{\mathrm{A}-\mathrm{S}}$, the corrugation of h-BN, the possibly relaxed distance between the top two nickel 

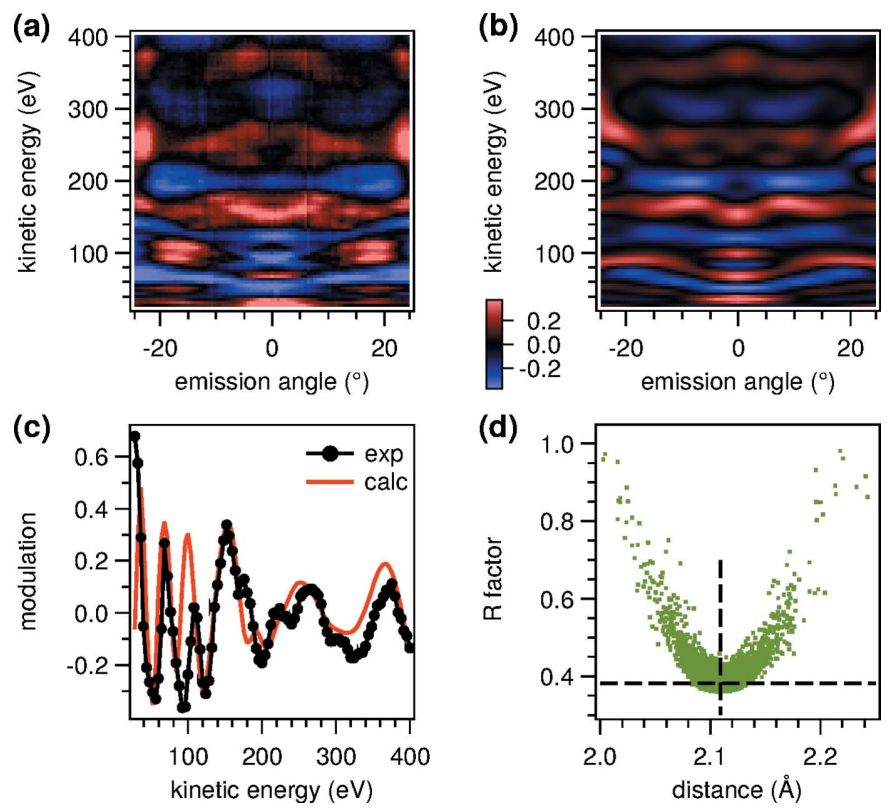

Figure 10

Energy-scanned photoelectron diffraction of the N $1 s$ peak of h-BN/ $\mathrm{Ni}(111)$ measured in backscattering geometry. (a) Two-dimensional experimental modulation function. The horizontal scale is the polar emission angle in the [1112] azimuth. (b) Calculated best fit modulation function. (c) One-dimensional modulation functions extracted from the experimental and calculated two-dimensional datasets at normal emission. (d) $R$-factor distribution versus adsorbate-substrate distance.

layers, the size of the cluster, the position of the refractive surface above the top layer, and the amplitude of the modulation function.

The measured modulation function normalized according to equation (1) is shown in Fig. 10, panels $(a)$ and (c). Panel (a) shows the full two-dimensional dataset $\chi\left(E_{\mathrm{kin}}, \alpha\right)$ while panel (c) shows a line profile $\chi\left(E_{\text {kin }}\right)$ integrated over $-2.5^{\circ}<\alpha<2.5^{\circ}$. The corresponding simulations of the optimized model structure are shown in panels $(b)$ and $(c)$. Panel $(d)$ shows the $R$-factor results from over 10000 calculated configurations as a function of $d_{\mathrm{A}-\mathrm{S}}$, the main parameter of interest. It shows a strong dependence on the adsorption parameter where the minimum $R=0.36$ designates the best-fit value, and the width of the distribution can be used to estimate the uncertainty according to Booth et al. (1997). The result is $d_{\mathrm{A}-\mathrm{S}}=(2.11 \pm$ $0.02) \AA$.

Since the raw data of Fig. 10(a) were measured with a twodimensional detector, we have carried out the optimization procedure for the two-dimensional and one-dimensional datasets separately. As can be seen in panels $(a)$ and $(b)$, the agreement between the calculation and the experiment is not reached in every detail, and the absolute values of the $R$-factor are correspondingly large. Nevertheless, the locations of the minima of $d_{\mathrm{A}-\mathrm{S}}$ are compatible in both cases. The advantage of a two-dimensional dataset is that multiple angles are measured at the same time. In the present case, this allows for a (coarse) optimization of other parameters such as the relaxed distance between the top two nickel layers $(1.99 \pm 0.04) \AA$, which is not possible from the normal emission measurement alone because the back-scattering directions are off-normal.

We conclude this section with a brief discussion of the normalization procedure applied to the angle-scanned data in Fig. 9(c). Processing of angle-scanned XPD data from a twodimensional electron analyser is more complex than from conventional channeltron-based detectors because the measured angle distribution is modified by additional physical and instrumental effects (Greif et al., 2014). Such effects include the angular dependence of the differential photoionization cross section, the cross section of the illuminated and the analysed volume, as well as angular inhomogeneities of the electron lens and the detector (transmission function). Fig. 11(a) shows the detector image of the $\mathrm{N} 1 \mathrm{~s}$ peak at the normal emission setting of the manipulator. The distribution of photoelectrons has a pronounced dependence on the polar emission angle $\alpha$ that is extremely sensitive to the distance of the sample from the entrance lens of the analyser. Only after measuring the data presented here we found that the transmission function could be flattened significantly by more careful alignment of the beam, the sample and the focal point (a)

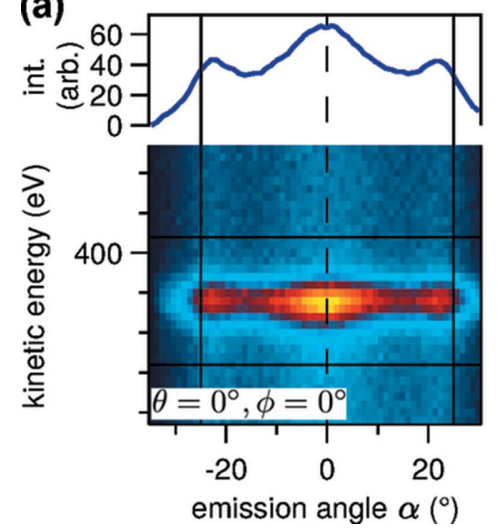

(b)

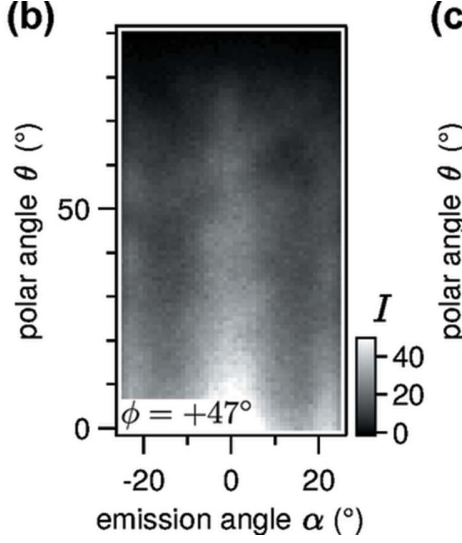

(c)

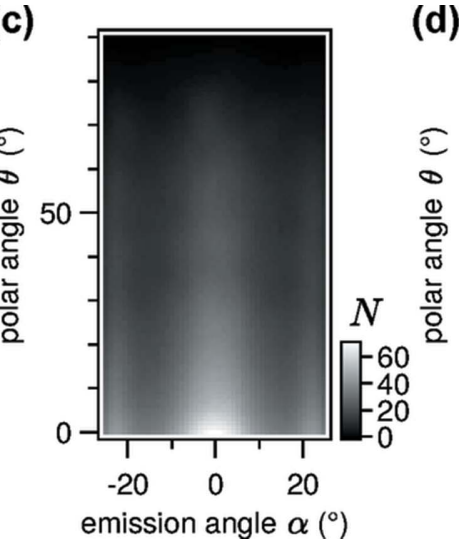

(d)

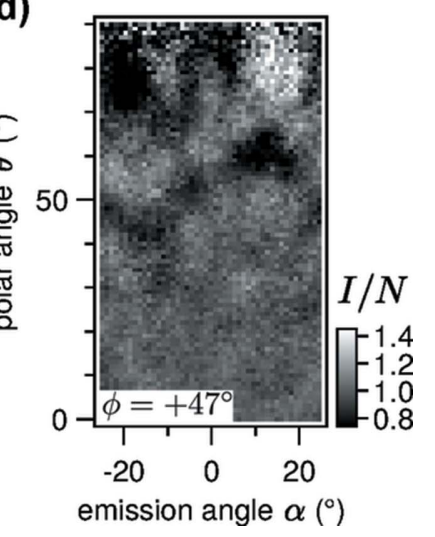

Figure 11

Normalization procedure of angle-scanned XPD data of h-BN/Ni(111). (a) Measured photoelectron intensity of the N $1 s$ peak versus kinetic energy and emission angle at the normal emission setting of the manipulator. The one-dimensional graph on top shows the integration over the full energy range. $(b)$ Polar angle scan $I(\alpha, \theta)$ at $\varphi=47^{\circ}$ after background subtraction and peak integration in the energy domain. $(c)$ Intensity distribution $I(\alpha, \theta)$ averaged over the 21 measured $\varphi$ positions. $(d)$ Polar scan from panel $(b)$ after normalization. 
of the analyser using a reference sample with well defined emission angles. However, a normalization step is still necessary in any case because the transmission curve is never perfectly flat and because of the polar dependence of the photoemission matrix element.

The normalization procedure is demonstrated on an excerpt from the raw data of the XPD measurement. A more detailed description is given in the supporting information. Fig. 11(b) shows the photoelectron intensity for a single polar scan trace at $\varphi=47^{\circ}$ after peak integration. The image contains diffraction features on top of the slowly varying, non-structural $\alpha$ and $\theta$ distribution. The normalization function $N(\alpha, \theta)$ is calculated by averaging $I(\alpha, \theta, \varphi)$ over all measured $\varphi$ settings and subsequent smoothing. Panel (c) shows that the diffraction features are washed out after averaging. This is most easily obtained if the azimuthal scan steps do not coincide with the symmetry of the sample. The normalization function is smoothed in $\alpha$ and $\theta$ using a locally weighted regression (LOESS) algorithm (Cleveland et al., 1992) with a smoothing factor large enough so that the smooth distribution varies slower than the diffraction features. By dividing $I / N$ we finally obtain the distribution shown in panel $(d)$. The features that are not related to diffraction have been successfully removed. The normalized distribution is finally mapped to the stereographic representation in Fig. 9(c).

\subsection{Quantum well states in a metal-organic network}

Metal-coordinated organic networks provide one possible route to integrate and connect molecular electronic devices with the help of self-assembly (Barth et al., 2005). In these networks, the spatial extent and the energetic alignment of the electronic states at the interface can be tuned by a judicious choice of molecular building blocks (Scheybal et al., 2009; Seufert et al., 2013; Wang et al., 2013). The mutual interaction of electronic states of adsorbate and substrate is, however, complex and poses a challenge to current numerical methods for theoretical predictions. Experimentally, the properties of occupied electronic states in ordered systems, including their degree of localization, are probed efficiently in ARPES (Lingle Jr et al., 1994; Lobo-Checa et al., 2009; Puschnig et al., 2011). STM and STS probe the local density of states directly, and are able to detect unoccupied states. Both technical features are helpful in the case of a two-dimensional metalorganic network of 9,10-dicyano-anthracene (DCA) molecules (Zhang et al., 2014). Grown by molecular beam deposition on a clean $\mathrm{Cu}(111)$ substrate at room temperature, this network exhibits a long-range periodic $8 \times 8$ porous superstructure as can be seen in the STM image in Fig. 12(a). The detailed topography image in panel $(b)$, measured after attaching a single DCA molecule to the STM tip, shows the arrangement of the molecules and the threefold coordination of the cyano groups with $\mathrm{Cu}$ adatoms with submolecular resolution.

$\mathrm{d} I / \mathrm{d} V$ spectra of the clean and DCA covered $\mathrm{Cu}(111)$ surface are shown in Fig. 12(c). The kink in the clean spectrum marks the onset of the Shockley surface state at $0.43 \mathrm{eV}$ below
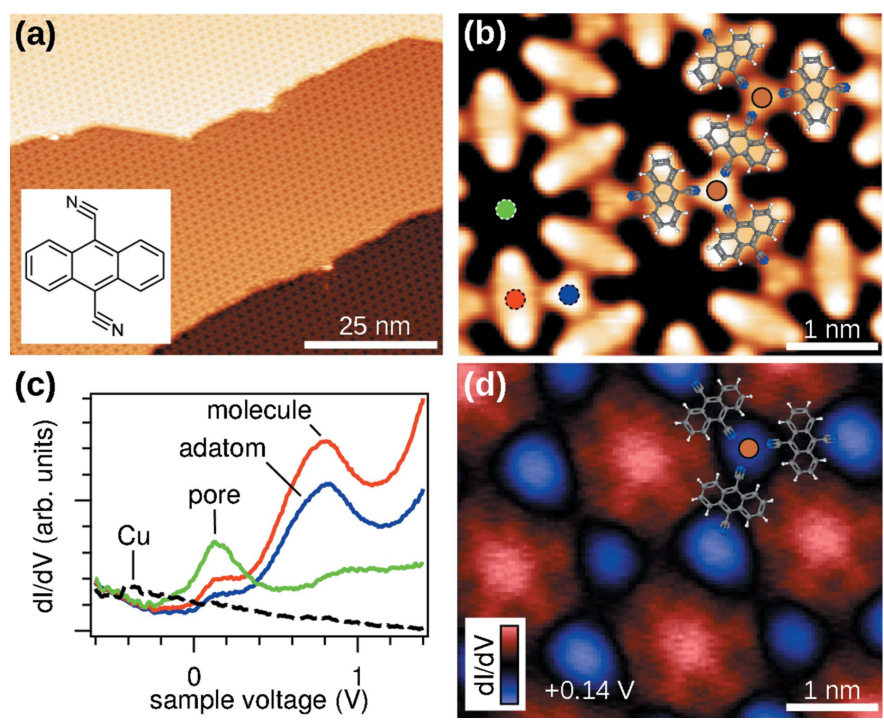

Figure 12

Self-assembled porous network of DCA molecules on $\mathrm{Cu}(111)$. (a) Widearea STM topography scan $(+2.0 \mathrm{~V}, 50 \mathrm{pA}, 300 \mathrm{~K})$. The inset shows the structural formula of DCA. (b) High-resolution STM topography image recorded with a DCA molecule attached to the STM tip $(-0.5 \mathrm{~V}, 100 \mathrm{pA}$, $4.4 \mathrm{~K}$ ). An (approximate) model of the molecular structure is overlaid. (c) $\mathrm{d} I / \mathrm{d} V$ spectra (metal tip, open feedback loop) at the positions marked in panel $(b)$ (solid curves), and on clean $\mathrm{Cu}(111)$ (dashed curve). Positive voltage corresponds to unoccupied states. $(d) \mathrm{d} I / \mathrm{d} V$ map at $+0.14 \mathrm{~V}$ showing the lateral distribution of the QWS (metal tip, open feedback loop). The image is slightly slanted due to a small drift during the scan which takes several hours.

the Fermi level. In strong contrast, the spectra of the DCA network, probed at different sites in the unit mesh, show distinct peaks of unoccupied states. Based on the site dependence of their amplitude we assign the peak at $+0.8 \mathrm{eV}$ to the molecular lattice, and the peak at $+0.14 \mathrm{eV}$ to a surface quantum well state (QWS) inside the pore (Zhang et al., 2014). The confined spatial distribution of the QWS peak becomes evident in a constant-height $\mathrm{d} I / \mathrm{d} V$ map at $+0.14 \mathrm{~V}$ bias in panel $(d)$.

Quantum well states are a result of the confinement of a dispersive state, in this case the free electron-like Shockley surface state of $\mathrm{Cu}(111)$, in a potential well imposed by an atomic structure of lower dimensionality (Crommie et al., 1993; Bürgi et al., 1998; Baumberger et al., 2002; Seufert et al., 2013). The confinement can be treated in the same way as the quantum mechanical particle in a box. The states inside the pore have to fulfill both the quadratic dispersion relation of the surface state, $E(k)=\hbar^{2} k^{2} / 2 m^{\star}$, and the boundary conditions of the quantum well which allow only a discrete series of states. The allowable wavevectors $k_{n}$ are given essentially by the reciprocal area of the quantum well ( $\mathrm{Li}$ et al., 1998; Kaufman et al., 1999). Since larger pores are present in small concentration at domain boundaries of the DCA network, states with different wavevectors can be probed with STS as illustrated in Fig. 13. Panel (a) shows that the first-order peak in the largest pore $A$ appears at a lower energy than the corresponding peak in the smaller pores $B$ and $C$. In addition to the first-order peak measured in all pores, second-order 

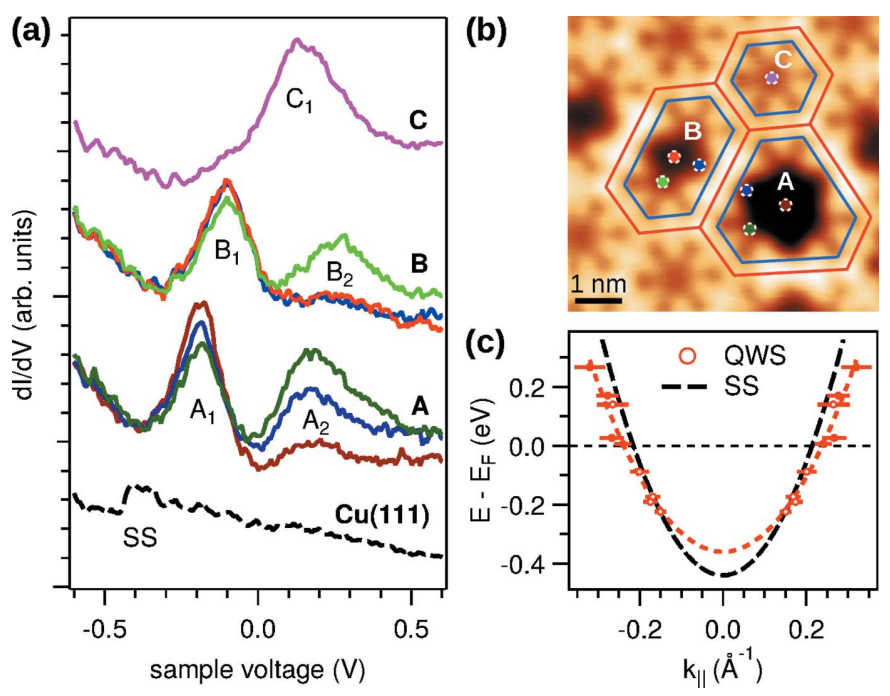

Figure 13

Quantum well states in the pores of the DCA network. (a) $\mathrm{d} I / \mathrm{d} V$ spectra of QWS in pores $A-C$. The dashed black curve is a reference spectrum measured on $\mathrm{Cu}(111)$, the band bottom of the surface state is labeled SS. (b) Topography image of three different sizes of pores at a $\mathrm{Y}$ intersection of domain boundaries $(-1.0 \mathrm{~V}, 50 \mathrm{pA}, 4.4 \mathrm{~K})$. Blue and red lines, respectively, indicate the boundary of the estimated and maximum area of the quantum well. Dots indicate the probed locations in panel $(a)$. (c) Energy dispersion of the quantum well states. Red dots are deduced from the peak position and pore size measured in a series of STS and STM measurements. Error bars indicate the uncertainty of determining the size of the pore. The fine dashed curve is a parabolic fit through the data points. The broken curve is the dispersion of the Shockley surface state on bare $\mathrm{Cu}(111)$ for reference.

peaks are observed in the larger pores $A$ and $B$. Using the estimated effective area of the quantum wells [blue boundaries in panel (b)], the dispersion of the QWS is plotted in panel $(c)$. We notice that, with respect to the unperturbed surface state, the dispersion of the QWS is shifted by $80 \mathrm{meV}$, and the effective mass is slightly (but not significantly) increased. We interpret the shift as a result of the overlap of the wavefunction with the finite confining barrier imposed by the molecular network (Zhang et al., 2014). This way, QWS can be used as a sensitive probe of the potential landscape in molecular adsorbate systems.

\subsection{Circular dichroism in photoelectron diffraction}

A bending magnet produces a superposition of linearly and circularly polarized synchrotron radiation. In the deflection plane, the light is linearly polarized, whereas the light emitted out of the plane contains a significant fraction of circularly polarized light. At the PEARL beamline, the trajectory of the stored electron beam inside the bending magnet can be tilted to extract partially polarized light in the same way as introduced earlier at the PolLux beamline X07DA (Raabe et al., 2008; Dunn et al., 2004).

Circularly polarized radiation is often used to study ordered magnetic moments in atomic systems due to $\mathrm{X}$-ray magnetic circular dichroism. Furthermore, circular dichroism in the angular distribution of photoelectrons has also been observed for non-magnetic systems. In Fig. 14, we demonstrate the

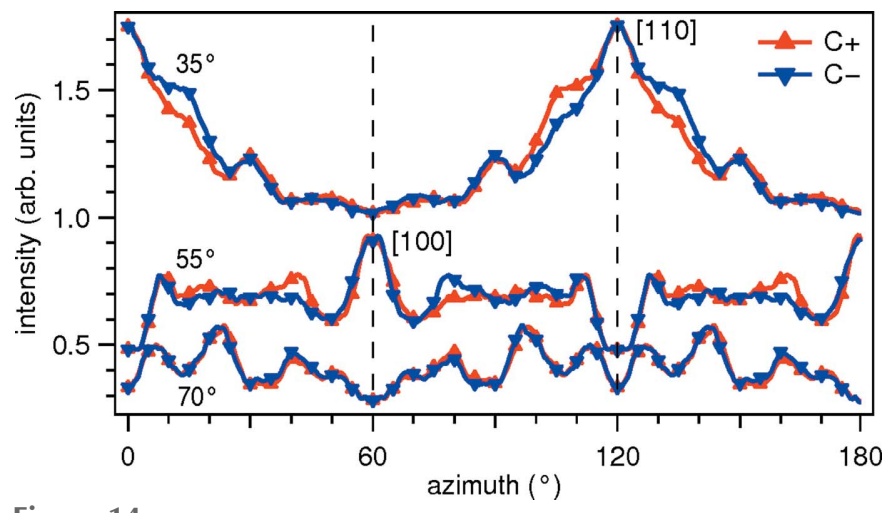

Figure 14

Angle-scanned photoelectron diffraction of the $\mathrm{Cu} 3 p_{3 / 2}$ photoelectron peak of a $\mathrm{Cu}(111)$ surface measured with left- $(\mathrm{C}-)$ and right-handed $(\mathrm{C}+)$ circularly polarized light at $h v=679.5 \mathrm{eV}$ and $E_{\text {kin }}=600 \mathrm{eV}$. Three pairs of azimuthal distribution curves are measured at polar angles of $35^{\circ}$, $55^{\circ}$ and $70^{\circ}$, cutting across the [110], the [100] and an arbitrary crystal direction, respectively. The curves are symmetrized for a slight difference in the degree of polarization at the two beamline settings. Sparse markers are drawn at every tenth data point.

transfer of angular momentum from the circularly polarized photon to the emitted photoelectron, which gives rise to a parallax shift of the forward-focusing peak in the angular distribution of the photoelectron intensity (Daimon, 2001). The curves show the integrated area of the $\mathrm{Cu} 3 p_{3 / 2}$ photoelectron peak as a function of the azimuthal rotation angle $\varphi$ (cf. Fig. 4) at three specific polar angles $\theta$. In the scan at $\theta=$ $35^{\circ}$, the peak and shoulder pattern at $\varphi=120^{\circ}$ is attributed to the forward-focusing of the photoelectron along the [110] direction (the nearest-neighbor direction in the $\mathrm{Cu}$ f.c.c. crystal). While the photoelectrons excited by linearly polarized photons are detected exactly at $120^{\circ}$, the photoelectrons which were excited by a circularly polarized photon deviate from the straight path and give rise to the shoulders at either side of the [110] direction, depending on the helicity of the photon. The effect is also seen in the [100] direction which corresponds to the second-nearest neighbor direction. Since they depend on the distance between the emitting and the scattering atom, such forward-focusing parallax shifts can be used as a means to measure interatomic distance (Daimon, 2001). If the atomic geometry is known, the variation of the forward focusing peak intensity can reveal site-specific local electronic and magnetic information (Matsui et al., 2008, 2015).

\section{Summary}

The performance measurements and the scientific examples show that the PEARL beamline of the Swiss Light Source is equipped for a wide range of surface science problems which can benefit from a combination of complementary experimental techniques. In particular, atomic structure can be studied with both local and space-averaging techniques. PEARL is one of very few synchrotron beamlines world-wide that are dedicated to photoelectron diffraction in angle- and energy-scanned modes. Because the instrumentation is mostly the same as for most photoemission spectroscopy methods, the 
beamline also supports the spectroscopy of core levels, resonant excitations, Auger modes or valence bands. The high resolution and stable imaging over several hours demonstrate the successful implementation of a low-temperature scanning tunneling microscope at a synchrotron facility. PEARL is open to users from the surface science community. Proposals are accepted semi-annually during the regular calls of the Swiss Light Source.

\section{Acknowledgements}

The authors would like to thank all scientists, engineers and technicians who have supported the project and contributed to the development of the beamline, especially (in alphabetical order) P. Ascher, Q. Chen, R. Follath, O. Groening, M. Mühlebach, C. Hess, A. Jaggi, J. Krempasky, L. H. de Lima, P. Müller, F. Nolting, J. Raabe, L. Rotach, V. Strocov, J. F. Van der Veen, and R. Wullschleger. Partial external funding from the Swiss National Science Foundation (R'EQUIP 206021121344) is gratefully acknowledged. JZ acknowledges funding by the European commission under the PSI-FELLOW/ COFUND programme, the Swiss National Science Foundation, the Swiss Federal Laboratories for Materials Science and Technology (Empa), the Swiss Nanoscience Institute (SNI) and the Université de Fribourg.

\section{References}

Auwärter, W., Kreutz, T. J., Greber, T. \& Osterwalder, J. (1999). Surf. Sci. 429, 229-236.

Auwärter, W., Muntwiler, M., Osterwalder, J. \& Greber, T. (2003). Surf. Sci. 545, L735-L740.

Barth, J. V. (2007). Annu. Rev. Phys. Chem. 58, 375-407.

Barth, J. V., Costantini, G. \& Kern, K. (2005). Nature (London), 437, 671-679.

Baumberger, F., Greber, T., Delley, B. \& Osterwalder, J. (2002). Phys. Rev. Lett. 88, 237601.

Berthold, W., Rebentrost, F., Feulner, P. \& Höfer, U. (2004). Appl. Phys. Mater. Sci. Process. 78, 131-140.

Booth, N. A., Davis, R., Toomes, R., Woodruff, D. P., Hirschmugl, C., Schindler, K.-M., Schaff, O., Fernandez, V., Theobald, A., Hofmann, P., Lindsay, R., Gießel, T., Baumgärtel, P. \& Bradshaw, A. M. (1997). Surf. Sci. 387, 152-159.

Bürgi, L., Jeandupeux, O., Hirstein, A., Brune, H. \& Kern, K. (1998). Phys. Rev. Lett. 81, 5370-5373.

Chen, C. T. \& Sette, F. (1989). Rev. Sci. Instrum. 60, 1616-1621.

Cleveland, W. S., Grosse, E. \& Shyu, M.-J. (1992). Software for locally weighted regression, http://www.netlib.org/a/dloess.

Corso, M., Verstraete, M. J., Schiller, F., Ormaza, M., Fernández, L., Greber, T., Torrent, M., Rubio, A. \& Ortega, J. E. (2010). Phys. Rev. Lett. 105, 016101.

Crommie, M. F., Lutz, C. P. \& Eigler, D. M. (1993). Science, 262, 218 220.

Daimon, H. (2001). Phys. Rev. Lett. 86, 2034-2037.

Despont, L., Koitzsch, C., Clerc, F., Garnier, M. G., Aebi, P., Lichtensteiger, C., Triscone, J.-M., Garcia de Abajo, F. J., Bousquet, E. \& Ghosez, P. (2006). Phys. Rev. B, 73, 094110.

Duncan, D. A., Choi, J. I. J. \& Woodruff, D. P. (2012). Surf. Sci. 606, 278-284.

Dunn, J. H. (2004). AIP Conf. Proc. 705, 65-68.

Fadley, C. S. (1984). Prog. Surf. Sci. 16, 275-388.

Fadley, C. S. (2010). J. Electron Spectrosc. Relat. Phenom. 178-179, $2-32$.
Fasel, R., Aebi, P., Agostino, R. G., Naumović, D., Osterwalder, J., Santaniello, A. \& Schlapbach, L. (1996). Phys. Rev. Lett. 76, 47334736.

Fasel, R., Wider, J., Quitmann, C., Ernst, K.-H. \& Greber, T. (2004). Angew. Chem. Int. Ed. 43, 2853-2856.

Föhlisch, A., Feulner, P., Hennies, F., Fink, A., Menzel, D., SanchezPortal, D., Echenique, P. M. \& Wurth, W. (2005). Nature (London), 436, 373-376.

Gamou, Y., Terai, M., Nagashima, A. \& Oshima, C. (1997). Sci. Rep. Res. Inst. Tohoku Univ. A44, 211-214.

García de Abajo, F. J., Van Hove, M. A. \& Fadley, C. S. (2001). Phys. Rev. B, 63, 075404.

Grad, G. B., Blaha, P., Schwarz, K., Auwärter, W. \& Greber, T. (2003). Phys. Rev. B, 68, 085404.

Greber, T., Šljivančanin, Ž., Schillinger, R., Wider, J. \& Hammer, B. (2006). Phys. Rev. Lett. 96, 056103.

Greif, M., Castiglioni, L., Becker-Koch, D., Osterwalder, J. \& Hengsberger, M. (2014). J. Electron Spectrosc. Relat. Phenom. 197, 30-36.

Jaouen, T., Razzoli, E., Didiot, C., Monney, G., Hildebrand, B., Vanini, F., Muntwiler, M. \& Aebi, P. (2015). Phys. Rev. B, 91, 161410.

Kato, M., Morishita, Y., Oura, M., Yamaoka, H., Tamenori, Y., Okada, K., Matsudo, T., Gejo, T., Suzuki, I. \& Saito, N. (2007). J. Electron Spectrosc. Relat. Phenom. 160, 39-48.

Kaufman, D. L. (1999). Am. J. Phys. 67, 133-141.

Kreutz, T. J., Greber, T., Aebi, P. \& Osterwalder, J. (1998). Phys. Rev. $B, \mathbf{5 8}, 1300-1317$

Li, J., Schneider, W.-D., Berndt, R. \& Crampin, S. (1998). Phys. Rev. Lett. 80, 3332-3335.

Lingle, R. L. Jr, Padowitz, D. F., Jordan, R. E., McNeill, J. D. \& Harris, C. B. (1994). Phys. Rev. Lett. 72, 2243-2246.

Lobo-Checa, J., Matena, M., Müller, K., Dil, J. H., Meier, F., Gade, L. H., Jung, T. A. \& Stöhr, M. (2009). Science, 325, 300-303.

Matsui, F., Fujita, M., Ohta, T., Maejima, N., Matsui, H., Nishikawa, H., Matsushita, T. \& Daimon, H. (2015). Phys. Rev. Lett. 114, 015501.

Matsui, F., Matsushita, T., Kato, Y., Hashimoto, M., Inaji, K., Guo, F. Z. \& Daimon, H. (2008). Phys. Rev. Lett. 100, 207201.

Meyer, M., Stähler, J., Kusmierek, D. O., Wolf, M. \& Bovensiepen, U. (2008). Phys. Chem. Chem. Phys. 10, 4932-4938.

Morscher, M., Nolting, F., Brugger, T. \& Greber, T. (2011). Phys. Rev. $B, \mathbf{8 4}, 140406$.

Muntwiler, M., Auwärter, W., Baumberger, F., Hoesch, M., Greber, T. \& Osterwalder, J. (2001). Surf. Sci. 472, 125-132.

Muntwiler, M., Auwärter, W., Seitsonen, A. P., Osterwalder, J. \& Greber, T. (2005). Phys. Rev. B, 71, 121402.

Oberta, P., Flechsig, U., Muntwiler, M. \& Quitmann, C. (2011). Nucl. Instrum. Methods Phys. Res. A, 635, 116-120.

Osterwalder, J., Aebi, P., Fasel, R., Naumović, D., Schwaller, P., Kreutz, T., Schlapbach, L., Abukawa, T. \& Kono, S. (1995). Surf. Sci. 331-333, 1002-1014.

Pawlak, R., Fremy, S., Kawai, S., Glatzel, T., Fang, H., Fendt, L.-A., Diederich, F. \& Meyer, E. (2012). ACS Nano, 6, 63186324.

Pawlak, R., Marot, L., Sadeghi, A., Kawai, S., Glatzel, T., Reimann, P., Goedecker, S., Güntherodt, H.-J. \& Meyer, E. (2015). Sci. Rep. 5, 13143.

Petersen, H., Jung, C., Hellwig, C., Peatman, W. B. \& Gudat, W. (1995). Rev. Sci. Instrum. 66, 1-14.

Piamonteze, C., Flechsig, U., Rusponi, S., Dreiser, J., Heidler, J., Schmidt, M., Wetter, R., Calvi, M., Schmidt, T., Pruchova, H., Krempasky, J., Quitmann, C., Brune, H. \& Nolting, F. (2012). J. Synchrotron Rad. 19, 661-674.

Puschnig, P., Reinisch, E.-M., Ules, T., Koller, G., Soubatch, S., Ostler, M., Romaner, L., Tautz, F. S., Ambrosch-Draxl, C. \& Ramsey, M. G. (2011). Phys. Rev. B, 84, 235427.

Raabe, J., Tzvetkov, G., Flechsig, U., Böge, M., Jaggi, A., Sarafimov, B., Vernooij, M. G. C., Huthwelker, T., Ade, H., Kilcoyne, D., 
Tyliszczak, T., Fink, R. H. \& Quitmann, C. (2008). Rev. Sci. Instrum. 79, 113704.

Reinert, F., Nicolay, G., Schmidt, S., Ehm, D. \& Hüfner, S. (2001). Phys. Rev. B, 63, 115415.

Scheybal, A., Müller, K., Bertschinger, R., Wahl, M., Bendounan, A., Aebi, P. \& Jung, T. A. (2009). Phys. Rev. B, 79, 115406.

Scheybal, A., Ramsvik, T., Bertschinger, R., Putero, M., Nolting, F. \& Jung, T. (2005). Chem. Phys. Lett. 411, 214-220.

Schillinger, R., Sljivančanin, Ž., Hammer, B. \& Greber, T. (2007). Phys. Rev. Lett. 98, 136102.

Seufert, K., Auwärter, W., García de Abajo, F. J., Ecija, D., Vijayaraghavan, S., Joshi, S. \& Barth, J. V. (2013). Nano Lett. 13, 6130-6135.

Strocov, V. N., Schmitt, T., Flechsig, U., Schmidt, T., Imhof, A., Chen, Q., Raabe, J., Betemps, R., Zimoch, D., Krempasky, J., Wang, X., Grioni, M., Piazzalunga, A. \& Patthey, L. (2010). J. Synchrotron Rad. 17, 631-643.
Treier, M., Ruffieux, P., Fasel, R., Nolting, F., Yang, S., Dunsch, L. \& Greber, T. (2009). Phys. Rev. B, 80, 081403.

Wang, S., Wang, W., Tan, L. Z., Li, X. G., Shi, Z., Kuang, G., Liu, P. N., Louie, S. G. \& Lin, N. (2013). Phys. Rev. B, 88, 245430.

Westerström, R., Dreiser, J., Piamonteze, C., Muntwiler, M., Weyeneth, S., Brune, H., Rusponi, S., Nolting, F., Popov, A., Yang, S., Dunsch, L. \& Greber, T. (2012). J. Am. Chem. Soc. 134, 98409843.

Westerström, R., Dreiser, J., Piamonteze, C., Muntwiler, M., Weyeneth, S., Krämer, K., Liu, S.-X., Decurtins, S., Popov, A., Yang, S., Dunsch, L. \& Greber, T. (2014). Phys. Rev. B, 89, 060406.

Wider, J., Greber, T., Wetli, E., Kreutz, T. J., Schwaller, P. \& Osterwalder, J. (1998). Surf. Sci. 417, 301-310.

Woodruff, D. P. (2007). Surf. Sci. Rep. 62, 1-38.

Zhang, J., Shchyrba, A., Nowakowska, S., Meyer, E., Jung, T. A. \& Muntwiler, M. (2014). Chem. Commun. 50, 12289-12292. 